\title{
Facteurs de stress psychologique et qualité du sperme dans une population de 450 hommes infertiles slovènes
}

\author{
B. ZORN, I. VIRANT-KLUN, M. KOLBEZEN, V. VELIKONJA, H. MEDEN-VRTOVEC \\ Centre d'Andrologie et service d'Aide Médicale à la Procréation, Clinique gynécologique \\ universitaire, Ljubljana, Slovénie
}

\section{RÉSUMÉ}

Le but de cette étude prospective a été d'examiner les relations entre divers facteurs de stress et la fonction de reproduction masculine dans deux populations : 450 hommes consultant pour infertilité du couple (groupe 1) et 45 hommes issus de couples en cours de traitement par AMP (groupe 2). Au cours du suivi des hommes, différentes caractéristiques psychologiques ont été étudiées par deux questionnaires, l'un dépistant la symptomatologie dépressive (questionnaire "WHO (five) Well-Being Index") et l'autre, le degré d'anxiété (questionnaire de ZUNG), complétés par des questions relatives à la réaction face au stress chronique quotidien et à une éventuelle exposition à un stress aigu. L'ensemble de ces données a été confronté aux résultats du spermogramme réalisé concomitamment. Dans le groupe 1, le trait dépressif (score $\leq 5$ ) était présent pour 6,5\% des hommes ; dans le groupe deux, 5,3\% des hommes étaient concernés $(4,8 \%$ dans le sousgroupe ICSI, 5,9 \% dans le sous-groupe FIV). Le trait anxieux (score $\geq 34$ ) était présent pour $20,3 \%$ des hommes du groupe 1 contre $30,8 \%$ des hommes du groupe 2 (50\% dans le sous-groupe ICSI contre $6 \%$ dans le sousgroupe FIV, p< 0,005). L'incidence des traits dépressifs et anxieux était deux fois plus élevée que celle observée dans une population générale. Tenant compte des facteurs de confusion (âge, durée d'abstinence sexuelle, rang de l'infertilité, notion de fièvre, antécé- dents de cryptorchidie et d'infection, varicocèle), les relations entre les valeurs des caractéristiques du sperme et les scores de dépression et d'anxiété, la réaction face au stress chronique et l'exposition à un stress aigu ont été étudiées par régression linéaire multiple. Il existait une relation positive entre le score de dépression et la concentration de spermatozoïdes (hausse de 7,3\% par unité de score), une relation négative entre la réaction face au stress chronique de la vie quotidienne, le nombre total des spermatozoïdes (baisse de 3,1 \% par unité de score), les mobilités progressives de type " $a$ " et " $a+b$ " et la vitalité (respectivement baisse de $0,5,0,5$ et $0,6 \%$ par unité de score) et une relation positive entre l'exposition au stress aigu et la mobilité “a+b"(hausse de 3,5\% en cas d'exposition à un stress aigu). Il n'a pas été retrouvé de différence pour les scores de dépression, anxiété, stress chronique et aigu selon qu'une grossesse spontanée ou non avait été observée. La présente étude indique que dans une population d'hommes en cours d'investigation ou de traitement pour une infertilité, la dépression

Correspondance : Dr. B. Zorn, Centre d'Andrologie et service d'Aide Médicale à la Procréation, Clinique gynécologique universitaire, Slajmerjeva 3, 1000 - Ljubljana Slovénie

e-mail : Milica.trenkic@guest.arnes.si

Communication orale sélectionnées au XVIIème Congrès de la SALF, 7-8 décembre 2000, Bordeaux. 
et l'anxiété sont fréquentes tandis que les caractéristiques du sperme peuvent être modulées de manière non univoque en fonction de la présence de dépression (baisse de la concentration des spermatozoïdes), de la réaction face au stress chronique (baisse du nombre, des mobilités " $a$ " et " $a+b$ " et de la vitalité) et de l'exposition au stress aigu (meilleure mobilité "a+b"). Ces résultats plaident en faveur d'une approche multidisciplinaire du couple et de l'homme inféconds avec prise en charge particulière de la dépression et de la réaction face au stress.

Mots-clés : anxiété, caractéristiques du sperme, dépression, hommes infertiles, stress aigu, stress chronique

\section{INTRODUCTION}

La qualité du sperme serait en baisse [1]. Délétères seraient en particulier les perturbateurs hormonaux [33]. L’idée que le stress psychologique influerait négativement la qualité $\mathrm{du}$ sperme repose essentiellement sur des études expérimentales chez l'animal $[6,8]$ et quasi expérimentales chez l'homme [34].

Les facteurs psychologiques liés au stress sont nombreux : stress chronique de la vie quotidienne, stress aigu et important - maladie et mort de proche [10], évènements dramatiques [12], "épreuves" de la fécondation in vitro (FIV) [31]. Ils auraient une influence négative sur les paramètres classiques du sperme selon des mécanismes dans lesquels serait impliqué l'axe hypothalamus-hypophyse-gonades. Dans l'étude rétrospective de 2343 spermogrammes chez des jeunes Slovènes, nous avions constaté une baisse de la mobilité dans la fin des années 80 faisant évoquer entre autres le stress psychologique, en rapport avec les évènements sociopolitiques, comme facteur responsable de cette détérioration [39]. Par ailleurs, au cours d'un stress important (épisode de la guerre d'indépendance en 1991), nous avions pareillement observé une baisse de la mobilité des spermatozoïdes [38].

Bien que moins connus que chez la femme, les facteurs psychologiques et leur retentissement sur la fertilité ont fait cependant l'objet de nombreuses études chez l' homme $[3,13,14$,
15]. La plupart cependant ne concerne qu'un groupe particulier d'hommes, par exemple hommes de consultation [20] ou hommes en cours de FIV [19, 24] ou donneurs de sperme [29], en général en nombre restreint et considérant le stress quotidien ou celui induit par le traitement de l'infertilité [30]. Par ailleurs, les études s'arrêtent aux facteurs de sperme sans s'intéresser au retentissement éventuel des modifications du spermogramme induites par le stress sur la survenue d'une grossesse spontanée.

Les buts de l'étude ont été d'étudier dans deux groupes distincts d'hommes infertiles (hommes vus en consultation à distance du traitement de procréation médicalement assistée (AMP) et hommes examinés en cours d'AMP), l'incidence des facteurs de stress psychologique et leur influence sur la qualité du sperme et sur la survenue d'une grossesse spontanée.

\section{MATÉRIEL ET MÉTHODES}

\section{Populations étudiées}

Entre le $1^{\mathrm{er}}$ novembre 1999 et le 31 octobre 2000, 1297 hommes appartenant à des couples inféconds sont venus au laboratoire d'andrologie pour effectuer un premier spermogramme. Selon l'OMS, est défini comme infécond tout couple pour lequel aucune grossesse n'a été observée après un an de rapports sexuels non protégés. Parmi ces hommes tous invités à participer à cette étude prospective, un consentement a été obtenu pour 450 (35\%) d'entre eux (groupe 1). Six mois après la réalisation de l'examen de sperme, ces hommes ont été contactés afin de savoir si une grossesse était survenue naturellement. En outre et au cours de la même période, l'étude a été proposée à un second groupe d'hommes appartenant à des couples en cours de traitement par AMP, FIV ou ICSI. Parmi ceux-ci, 45 hommes (FIV, $\mathrm{n}=21$, ICSI, $n=24$ ) ont accepté de participer à l'étude (groupe 2). Les caractéristiques du sperme le jour de la ponction ovocytaire ont été enregistrées. Lindication de la FIV était exclusivement le facteur tubaire. Les indications de l'ICSI étaient l'oligoasthénotératozoospermie (OAT) dans 19 cas, une tératozoospermie isolée (1 cas) et un échec de fécondation en FIV malgré un spermogramme normal. 


\section{Spermogramme}

Le sperme était recueilli par masturbation au laboratoire après un délai d'abstinence sexuelle de 3 à 5 jours. L'analyse du sperme était faite selon les recommandations de l'OMS [36]. Dans le groupe 2 (patients en cours d'AMP), seules les caractéristiques suivantes ont été étudiées : volume de l'éjaculat, concentration, vitalité et mobilité " $a+b$ " des spermatozoïdes.

\section{Questionnaires}

Le jour du spermogramme pour le groupe 1 et le jour de la ponction ovocytaire dans le cas du groupe 2, chaque patient ayant donné son consentement pour l'étude a rempli un questionnaire portant sur les expositions professionnelles, le style de vie (consommation de tabac, d'alcool, notion de fièvre, etc...) et des questions se rapportant à l'exposition au stress et à l'état psychologique. En ce qui concerne l'évaluation du stress, une première série de questions avait trait à la réaction des hommes face à différentes situations stressantes de la vie quotidienne (stress chronique), liées aux conditions de travail, à la vie de couple, aux relations familiales, à la vie sexuelle, à l'état de santé et à la situation d'infécondité. Pour chacun de ces facteurs, un score de satisfaction basé sur une échelle de 1 à 7 , de la situation la moins satisfaisante à la situation la plus satisfaisante, était proposé. Statuant que chaque individu est exposé à un certain niveau de stress, la réaction face au stress est estimée d'autant plus forte que l'individu s'estime être plus satisfait de son état. Une deuxième série de questions portant sur des situations possiblement génératrices de stress aigu et important (perte d'emploi, divorce, déménagement, accident grave de la voie publique, maladie de l'homme, maladie et/ou mort d'un proche) dans les 3 mois précédant était posée (réponse par non $(=1)$ ou par oui $(=2)$ ). La mise en évidence des traits psychologiques des hommes pouvant moduler le stress a été faite à l'aide de deux questionnaires standardisés sur les traits psychologiques de dépression et d'anxiété : la version $1995 \mathrm{du}$ questionnaire WHO (five) WellBeing Index [37] (score entre 0 et 15) et le questionnaire de Zung, Zung's Anxiety Scale Inventory [2] (score entre 20 et 80 ), respectivement et il était demandé d'ajuster les réponses à la période des deux semaines précédentes. Dans l'échelle du WHO (five) Well-Being Index, un score total de 5 points et moins dépiste un trait pschychologique dépressif. Dans l'échelle de Zung, un score total de 34 et plus indique que la personne présente un trait de personnalité anxieuse.

\section{Examen clinique}

Chaque homme a fait l'objet d'un examen clinique, orienté sur la sphère urogénitale, dans le mois qui a suivi le spermogramme.

\section{Statistiques}

Les tests statistiques ont été réalisés à l'aide du logiciel SPSS (SPSS Inc., Illinois, USA, version 9).

La comparaison entre les traits dépressifs ou anxieux (valeur des scores) en fonction du type d'AMP (dans le groupe 2) a été faite par le test de Chi2.

Les relations entre les caractéristiques du sperme (pour le nombre total des spermatozoïdes, une transformation logarithmique (base 10) a été utilisée) et les traits psychologiques de dépression, d'anxiété et de réaction face au stress chronique (valeurs des scores) et l'exposition au stress aigu ont été analysées par régression linéaire multiple. Nous avons tenu compte des possibles facteurs de confusion : âge, durée d'abstinence sexuelle, rang d'infertilité, notion de fièvre, antécédents de cryptorchidie et d'infection génitale et présence de varicocèle.

Il a été recherché par le test de Mann-Whitney si des différences dans les scores de dépression, d'anxiété et de réaction face au stress chronique ou d'exposition à un stress aigu existaient selon qu'une grossesse spontanée ou non avait été obtenue dans les six mois suivant le prélèvement de sperme pour les 114 premiers hommes inclus dans l'étude.

\section{RÉSULTATS}

\section{Caractéristiques des hommes étudiés}

Dans le groupe 1, l'âge moyen des hommes était de 33 ans $( \pm 5,4$ ans), celui des partenaires de 31 ans $( \pm 4,7$ ans), l'infertilité était primaire dans $70,5 \%$ des cas, la durée moyenne d'infé- 
condité était de 3,4 ans ( $\pm 3,0$ ans), il existait $42 \%$ de facteurs féminins d'infertilité (tubaires majoritairement). Vingt et un hommes présentaient une azoospermie ( $4 \%$ ), 110 une oligo- et asthéno- et tératozoospermie $(24,4 \%)$.

Dans le groupe 2 (AMP), l'âge moyen des hommes était de 36 ans ( $\pm 6,0$ ans) et l'infertilité était primaire dans $59 \%$ des cas. Les caractéristiques du sperme dans les deux groupes sont présentées dans le Tableau 1.

\section{Traits psychologiques dépressif ou anxieux dans les deux groupes d'hommes étudiés}

Dans le groupe 1, le trait dépressif était présent pour 6,5\% des hommes ; dans le groupe 2 , $5,3 \%$ des hommes étaient concernés $(4,8 \%$ dans le sous-groupe ICSI, 5,9\% dans le sousgroupe FIV). Le trait anxieux était présent pour 20,3\% des hommes du groupe 1 contre $30,8 \%$ des hommes du groupe 2 (50\% dans le sous-groupe ICSI contre $6 \%$ dans le sous-groupe FIV, $p<0,005$ ).

\section{Caractéristiques du sperme et scores de dépression et d'anxiété, réaction face au stress chronique et exposition au stress aigu dans le groupe 1}

Les résultats significatifs de l'analyse en régression multiple sont présentés dans les tableaux 2 à 4 . La tendance dépressive (lorsque le score de dépression diminuait) était associée à une baisse de la concentration des spermatozoïdes (Tableau 2).

Plus la réaction face au stress chronique était importante, moindres étaient le nombre total des spermatozoïdes (Tableau 2), les mobilités "a" et "a+b" (Tableau 3) et la vitalité (Tableau 4). En cas d'exposition à un stress aigu, la mobilité " $a+b$ " était meilleure (Tableau 3).

\section{Survenue d'une grossesse et facteurs de stress}

Dans la période de 6 mois suivant le prélèvement de sperme et pour les 114 hommes étudiés, il y a eu 50 grossesses spontanées. Aucune différence significative pour les scores de dépression, d'anxiété, stress chronique et aigu n'a été trouvée selon qu'une grossesse ou non avait été observée.

\section{DISCUSSION}

Les hommes infertiles sont volontiers stressés, déprimés et anxieux [15] avec une perte de la masculinité et de l'estime de soi [23]. Lincidence de la symptomatologie dépressive et de l'anxiété chez nos patients de consultation et d'AMP est deux fois plus élevée que celle retrouvée dans la population générale (respectivement 3-4 et $11 \%$ ) [15, 37]. Il en est de même pour l'anxiété pour les deux groupes, consultation et AMP. Chez les patients d'AMP, tant femmes qu'hommes, l'anxiété est fortement exprimée [7]. La tentative de FIV est en effet vécue comme très stressante, tant chez la femme que chez l'homme. Ce n'est pas tant le côté physique de la FIV (exemple de la masturbation) que surtout l'attente et la révélation des résultats de la FIV et plus tard de la grossesse [24] qui stressent. La plus forte anxiété de nos patients ICSI par rapport à ceux de FIV classique n'est pas une surprise. À la différence de Boivin [4], d'autres auteurs l'ont également retrouvée [5]. Les hommes du groupe ICSI sont conscients qu'ils sont responsables de l'infertilité alors que leur partenaire est souvent indemne de toute lésion génératrice d'infécondité. Ils craignent que la tentative d'ICSI n'échoue justement à cause de leur sperme de mauvaise qualité et l'attente des résultats est encore plus dure à supporter. Par ailleurs, il y a la crainte de l'état physique et intellectuel de l'enfant à naître, éventuellement anormal en raison du mauvais sperme. Tout cela plaide en faveur de l'appui souvent nécessaire d'un psychologue au cours du traitement de la stérilité de couple et de la nécessité pour le médecin de donner au patient la juste information, toutes démarches qui diminueront l'anxiété et restaureront l'estime de soi et les chances de succès [15].

De nombreux facteurs liés à l'environnement incluant le stress psychologique influent négativement sur la qualité du sperme [25]. Les mécanismes en cause sont probablement multiples (directs et indirects au travers de l'abus d'alcool et de tabac et autres drogues) et notoirement mal connus, faisant intervenir les modifications hormonales de la prolactine et de la testostérone en particulier qui retentiraient sur la spermatogénèse $[11,13]$. Pour certains, 
Tableau 1 : Valeur moyenne ( \pm écart-type) des caractéristiques du sperme dans le groupe d'hommes consultant pour infécondité (groupe 1) et le groupe d'hommes issus de couples en cours d'AMP (groupe 2). ND = non déterminé

\begin{tabular}{lccc}
\hline Caractéristique & $\begin{array}{c}\text { Groupe 1 } \\
(\mathbf{n = 4 5 0})\end{array}$ & FIV (n=24) & ICSI (n=21) \\
Volume (ml) & $3,8 \pm 1,7$ & $1,7 \pm 0,9$ & $2,6 \pm 0,9$ \\
Concentration de & & $81,9 \pm 18,3$ & \\
spermatozoïdes $\left(\mathrm{x} 10^{6} / \mathrm{ml}\right)$ & $64,5 \pm 57,0$ & & $18,9 \pm 29,1$ \\
Nombre total de & & $166,4 \pm 69,5$ & $30,6 \pm 53,6$ \\
spermatozoïdes $\left(\times 10^{6}\right)$ & $238 \pm 216$ & $72 \pm 8$ & $39 \pm 30$ \\
\% spermatozoïdes & $49 \pm 15$ & $64 \pm 9$ & $42 \pm 7$ \\
mobiles "a+b" & $63 \pm 11$ & $\mathrm{ND}$ & $\mathrm{ND}$ \\
\% spermatozoïdes vivants & $27 \pm 16$ & & \\
\% spermatozoïdes normaux & & & \\
\end{tabular}

Tableau 2 : Relation entre la concentration et le nombre total des spermatozoïdes et les scores de dépression et d'anxiété, la réaction face au stress chronique et l'exposition au stress aigu par analyse en régression multiple (groupe 1)

$\begin{array}{lll}\text { Coefficients non standardisés } & \text { Coefficient } & \text { p } \\ \text { B } & \text { Erreur standard } & \text { standardisé } \beta\end{array}$

\section{CONCENTRATION}

Score de dépression

$4,74^{*} \quad 2,21$

0,182

0,03

Score d'anxiété$$
\text { - }
$$

Réaction face au

stress chronique

Exposition au stress aigu

$-$

\section{NOMBRE TOTAL DES}

\section{SPERMATOZOIDES}

Score de dépression

Score d'anxiété

Réaction face au stress

chronique

$-0,0136^{* * *}$

0,007

$-0,164$

NS

Exposition au stress aigu

$\begin{array}{llll}- & - & - & \text { NS } \\ - & - & - & \text { NS } \\ -0,0136^{* *} & 0,007 & -0,164 & 0,052 \\ - & - & - & \text { NS }\end{array}$

*La concentration augmente de $4,74.10^{6} \mathrm{spz} / \mathrm{ml}$ (soit 7,3\%) par unité de score

**L'anti-log de la valeur logarithmique du coefficient de régression indique que le nombre total des spermatozoïdes chute de $4,0.10^{6} \mathrm{spz}$ (soit $3,1 \%$ ) par unité de score 
Tableau 3 : Relation entre le pourcentage des spermatozoüdes mobiles " $a$ " et des spermatozoüdes mobiles " $a+b$ " et les scores de dépression et d'anxiété, la réaction face au stress chronique et l'exposition au stress aigu par analyse en régression multiple (groupe 1)

$\begin{array}{lll}\text { Coefficients non standardisés } & \begin{array}{l}\text { Coefficient } \\ \text { Btandardisé } \beta\end{array} & \text { p }\end{array}$

\section{SPERMATOZOIDES}

MOBHLES « $\mathbf{a}$ »

Score de dépression

Score d'anxiété

Réaction face au

stress chronique

$-0,522$

0,196

Exposition au stress aigu

SPERMATOZOIDES MOBILES « $a+b$ »

Score de dépression

Score d'anxiété

Réaction face au stress chronique

Exposition au stress aigu

$\begin{array}{ll}- & - \\ - & - \\ -0,516 & 0,208 \\ 3,495 & 1,782\end{array}$

1,782
$-0,217$

0,008

$\begin{array}{ll}- & \text { NS } \\ - & \text { NS } \\ -0,217 & 0,008 \\ - & \text { NS }\end{array}$

NS

NS

0,014

0,051

Tableau 4 : Relation entre le pourcentage de spermatozoüdes vivants et les scores de dépression et d'anxiété, la réaction face au stress chronique et l'exposition au stress aigu par analyse en régression multiple (groupe 1)

$\begin{array}{llll}\text { Coefficients non standardisés } & \text { Coefficient } & \text { p } \\ \text { B } & \text { Erreur standard } & \text { standardisé } \beta\end{array}$

Score de dépression

-
-
-

$\begin{array}{ll}- & \text { NS } \\ - & \text { NS }\end{array}$

Réaction face au stress

chronique

$-0,618$

0,183

$-0,272$

0,001

Exposition au stress aigu

-

(2)

NS 
il y aurait un fléchissement de la qualité du sperme chez les hommes immédiatement avant la FIV [15, 26], pouvant se traduire par une baisse du taux de fécondation [19]. Giblin [14] étudiant, chez 28 hommes sains volontaires, la qualité du sperme émis toutes les 2 semaines pendant 6 mois retrouve une corrélation négative entre les facteurs de stress et le volume et le pourcentage de morphologie normale. Cette détérioration du sperme en lien avec le stress n'existe pas pour Hammond [17] (ayant inclus des patients de FIV et d'insémination intra-utérine) ni pour Pellicer [28]. Nous avons retrouvé une influence négative des facteurs du stress sur la qualité de sperme, en particulier en présence d'une symptomatologie dépressive et d'une forte implication de l'homme face au stress quotidien.

Quelques études ont trait à la présence de la dépression chez les hommes infertiles et à son éventuel retentissement sur la qualité du sperme et la capacité de reproduction. Avant que l'homme ne consulte, il est surtout anxieux. Au décours de la consultation médicale, l'anxiété diminue et fait place à la dépression [15]. Dans un groupe AMP, l'incidence de la dépression est anormalement élevée [24]. Le groupe de Münster n'a pas retrouvé de modifications des paramètres du sperme selon que l'homme était déprimé ou non et conclut que la dépression n'influe pas sur le potentiel reproducteur de l'homme $[20,22]$. Nous avons mis en évidence une diminution de la concentration des spermatozoïdes tandis que le volume augmentait.

La réaction face au stress quotidien est primordiale. En effet, lorsque les patients prennent de la distance par rapport à leur problème d'infertilité, il y a amélioration des paramètres du sperme $[22,30]$. Dans notre expérience, une forte réaction de l'homme face au stress quotidien était pareillement liée à une influence négative sur le sperme.

En étudiant les relations entre stress et qualité de sperme chez des donneurs du sperme (étudiants en médecine), Poland [29] a mis en évidence un impact positif du stress - induit par les périodes d'examens - sur le sperme. Nous avons pareillement retrouvé une influence positive de l'exposition au stress aigu sur la mobilité des spermatozoïdes. Cette relation surprenante est peut-être à rattacher à l'effet bénéfique d'une certaine dose de stress sur l'organisme. On notera qu'à la différence de Fenster [10] qui retrouve une influence négative uniquement en cas de stress très important (mort de proche), nous n'avions pas de tel cas dans notre population.

L'anxiété, dans notre expérience, n'avait aucun lien avec la qualité du sperme. Dans un groupe AMP (sans distinction de la méthode - FIV ou ICSI - utilisée), Clarke [5] remarquait que l'anxiété corrélait avec une baisse de la qualité du sperme. Cependant, chez les patients ICSI cette relation disparaissait. Il rattachait cela au fait que le sperme était auparavant déjà très altéré et qu'il aurait été difficile de le voir s'altérer plus encore. Pour Hammond [17], la participation d'hommes anxieux, aux traitements de l'infertilité, n'avait pas d'influence négative sur la qualité du sperme. Chez certains patients anxieux, les paramètres du sperme s'étaient même améliorés. Boivin [4] non plus n'a pas retrouvé de baisse de qualité de sperme malgré un surcroît d'anxiété chez les patients ICSI un jour avant la ponction.

Limpact du stress sur la survenue d'une grossesse a été souvent étudié en particulier chez la femme en cours d'AMP. La baisse de l'anxiété [18], l'amélioration des paramètres psychologiques [32] avec moindre vulnérabilité aux facteurs de stress [9] sont liées à l'obtention d'une grossesse. Cependant une forte anxiété et un stress élevé n'induisent pas une issue défavorable de la grossesse en terme de fausses couches [27]. Après chaque échec d'AMP s'installe un véritable travail de deuil [7]. Enfin, l'intensité du désir d'enfant est corrélée au degré de stress. Pour Hubert [22], la revendication sociale et l'extraversion sont liées à l'infertilité masculine tandis que la régression, l'hypochondrie et la vacuité émotionnelle sont en relation avec de meilleures caractéristiques de fertilité. L’influence du stress sur la survenue d'une grossesse spontanée a été étudiée par Hjollund [21] qui ne retrouve pas de relation entre un environnement particulièrement stressant au travail et une réduction de la fertilité.

Nous n'avons pas retrouvé de différence dans les facteurs de stress entre les hommes fertiles 
et ceux infertiles. Probablement, cela est à rattacher à l'impact négatif ou positif relativement modéré des facteurs du stress sur les paramètres du sperme.

Notre étude confirme la forte incidence de la symptomatologie dépressive et de l'anxiété chez les hommes de couples infertiles. Une forte implication dans la réaction au stress et la symptomatologie dépressive ont une influence négative sur la qualité du sperme, l'anxiété non. Le stress aigu de moindre intensité peut résulter en une action stimulante.

C'est l'occasion de rappeler combien la prise en charge psychologique du couple et de l'homme [16] peut être souhaitable au cours de l'exploration et du traitement d'une infertilité. La prise en charge contribuera à améliorer les paramètres du sperme et à augmenter l'harmonie du couple qui est selon Stoleru [35] un préalable à une meilleure fécondation en FIV.

\section{REMERCIEMENTS}

Ce travail a été réalisé dans le cadre du projet PROTEUS, soutenu par les ministères français des Affaires étrangères et slovène des Sciences et de la technologie.

Nous remercions Ivan Verdenik pour l'analyse statistique, le personnel des consultations d'andrologie de la clinique gynécologique (en particulier mesdames Draga Plavc et Zdravka Petkovi) et tous les patients qui ont donné de leur temps et disponibilité pour faire avancer la science.

Nous remercions enfin le docteur Jacques Auger de nous avoir aidé pour l'analyse statistique et accompagnés de ses conseils pertinents.

\section{RÉFÉRENCES}

1. AUGER J., KUNSTMANN J., CZYGLIK F., JOUANNET P. : Decline in semen quality among fertile men in Paris during the past 20 years. N. Engl. J. Med., 1995, 332 : 281-285.

2. BECH P. : Rating Scales for psychopathology, health status and quality of life. A compendium on documentation in accordance with the DSM-III-R and WHO systems. Berlin, Springer-Verlag, 1993.

3. BIGELOW P.L., JARRELL J., YOUNG M.R., KEEFE T.J., LOVE E.J. : Association of semen quality and occupational factors: comparison of case-control ana- lysis and analysis of continuous variables. Fertil. Steril., 1998, 69 : 11-18.

4. BOIVIN J., SHOOG-SVANBERG A., ANDERSSON L. et al. Distress level in men undergoing intracytoplasmic sperm injection versus in-vitro fertilization. Hum. Reprod., 1998, 13 : 1403-1406.

5. CLARKE R.N., KLOCK S.C., GEOGHEGAN A., TRAVASSOS D.E. : Relationship between psychological stress and semen quality among in-vitro fertilization patients. Hum. Reprod., 1999, 14 : 753-758.

6. COCKETT A.T.K., ELBADAWI A., ZEMJANIS R., ADEY W.R. : The effects of immobilization on spermatogenesis in subhuman primates. Fertil. Steril., 1970, $21: 610-614$.

7. COLLINS A., FREEMAN E.W., BOXER A.S., TURECK R. : Perceptions of infertility and treatment stress in females as compared with males entering in vitro fertilization treatment. Fertil. Steril., 1992, 57, 350-356.

8. CUI K.-H. : The effect of stress on semen reduction in the marmoset monkey (Callithrix jacchus). Hum. Reprod., 1996, 11 : 568-573.

9. FACHINETTI F., MATTEO M.L., ARTINI G.P., VOLPE A., GENAZZANI A.R. : An increased vulnerability to stress is associated with a poor outcome of in vitro fertilization-embryo transfer treatment. Fertil. Steril., 1997, 67 : 309-314.

10. FENSTER L., KATZ D.F., WYROBEK A.J. et al. : Effects of psychological stress on human semen quality. J. Androl., 1997, 18 : 194-202.

11. FRANCIS K.T. : The relationship between high and low trait psychological stress, serum testosterone, and serum cortisol. Experientia, 1981, 37 : 1296-1297.

12. FUKUDA M., FUKUDA K., SHIMIZU T., YOMURA W., SHIMIZU S. : Kobe earth quake and reduced sperm motility. Hum. Reprod., 1996, 11 : 1244-1246.

13. GERHARD I., LENHARD K., EGGERT-KRUSE W., RUNNEBAUM B. : Clinical data which influence semen parameters in infertile men. Hum. Reprod., 1992, $7: 830-837$.

14. GIBLIN P.T., POLAND M.L., MOGHISSI K.S., AGER J.W., OLSON J.M. : Effects of stress and characteristic adaptability on semen quality in healthy men. Fertil. Steril., 1988, 49 : 127-132.

15. GLOVER I., GANNON K., SHERR L., ABEL P.D. : Distress in sub-fertile men : a longitudinal study. J. Reprod. Inf. Psychol., 1996, 14 : 23-36.

16. GLOVER I., ABEL P.D. : Male subfertility: is pregnancy the only issue? Lancet, 1998, $316: 1405-1406$.

17. HAMMOND K.R., KRETZER P.A., BLACKWELL R.E., STEINKAMPF M.P. : Performance anxiety during infertility treatment : effect on semen quality. Fertil. Steril., 1990, 53 : 337-340.

18. HARLOW C.R., FAHY U.M., TALBOT W.M., WARDLE P.G., HULL M.G.R. : Stress and stress-related 
hormones during in-vitro fertilization treatment. Hum. Reprod., 1996, 11 : 274-279.

19. HARRISON K.L., CALLAN V.J., HENNESSEY J.F. : Stress and semen quality in an in vitro fertilization program. Fertil. Steril., 1987, $48: 633-636$.

20. HELLHAMMER D.H., HUBERT W., FREISCHEM C.W., NIESCHLAG E. : Male infertility relationship among gonadotrophins, sex steroids, seminal parameters and personality attitudes. Psychosom. Med., 1985, $47: 58-66$.

21. HJOLLUND N.H., KOLD JENSEN T., BONDE J.P. et al. Job strain and time to pregnancy. Scand. J. Work Environ. Health, 1998, 24 : 344-350.

22. HUBERT W., HELLHAMMER D.H., FREISCHEM C.W. : Psychological profiles in infertile men. J. Psychosom. Res., 1985, 29 : 161-165.

23. KEDEM P., MIKULINCER M., NATHANSON Y.E., BARTOOV B: : Psychological aspects of male infertility. Br. J. Med. Psychol., 1990, 63 : 73-80.

24. KENTENICH H., SCHMIADY H., RADKE E., STIEF G., BLANKAU A. : The male IVF patient - psychosomatic considerations. Hum. Reprod., 1992, 7, suppl.1: 13-18.

25. MACLEOD J. : The significance of deviations in human sperm morphology. Adv. Exp. Biol. Med., 1970, $10: 481-494$.

26. MAHLSTEDT P.P. : Psychological issues of infertility and assisted reproductive technology. Urol. Clin. North Am., 1994, 21 : 557-566.

27. MILAD M.P., KLOCK S.C., MOSES S., CHATTERTON R. : Stress and anxiety do not result in pregnancy wastage. Hum. Reprod., 1998, $13: 2296-2300$.

28. PELLICER A., RUIZ M. : Fertilization in vitro of human oocytes by spermatozoa collected in different stressful situations. Hum. Reprod., 1989, 4 : 817-820.

29. POLAND M.L., GIBLIN P.T., AGER J.W., MOGHISSI K.S. Effect of stress on semen quality in semen donors. Int. J. Fertil., 1986, 31 : 229-231.

30. POOK M., KRAUSE W., ROHRLE B. : Coping with infertility: distress and changes in sperm quality. Hum. Reprod., 1999, 14 : 1487-1492.

31. RAGNI G., CACCAMO A. : Negative effect of stress of in vitro fertilization program on quality of semen. Acta Eur. Fertil., 1992, $23: 21-23$.

32. SANDERS K.A., BRUCE N.W. : A prospective study of psychosocial stress and fertility in women. Hum. Reprod., 1997, 12 : 2324-2329.

33. SHARPE R.M., SKAKKEBAEK N.E. : Are oestrogens involved in falling sperm counts and disorders of the male reproductive tract? Lancet, 1993, 314 : 13921395.

34. STIEVE H. : Der Einfluss des Nervensystems auf Bau und Tätigkeit der Geschlechtorgane des Menschen. Mit $93 \mathrm{Abb}$. von B. Neresheimer. Stuttgart, Thieme, 1952.
35. STOLERU S., CORNET D., VAUGEOIS P. et al. : The influence of psychological factors on the outcome of the fertilization step of in vitro fertilization. J. Psychosom. Obstet. Gynaecol., 1997, 18 : 189-202.

36. WORLD HEALTH ORGANIZATION : WHO laboratory manual for the examination of human semen and semen-cervical mucus interaction. 4th edn. Cambridge, UK, Cambridge University Press, 1999.

37. WORLD HEALTH ORGANIZATION. Mastering depression in primary care. Version 2.2 WHO (five) Well-Being Index. WHO Regional Office for Europe, 1999.

38. ZORN B., VIRANT-KLUN I., MEDEN-VRTOVEC H.: Stress induced by short-term war affected sperm motility in young men from Slovenia. In: Program and Abstract Book of Serono International Symposium on Male Sterility for Motility Disorders: Etiological factor and Treatment, 1998, January 30-31, ASIEM, Paris, France, P7.

39. ZORN B., VIRANT-KLUN I., VERDENIK I., MEDEN-VRTOVEC H. : Semen quality changes among Slovenian healthy men included in the IVF-ET programme during 1983-1996. Int. J. Androl., 1999, $22: 178-183$.

\section{ABSTRACT}

Psychological stress and sperm quality in $\mathbf{4 5 0}$ infertile Slovenian men

ZORN B., VIRANT-KLUN I., KOLBEZEN M.,
VELIKONJA V., MEDEN-VRTOVEC H.

The purpose of this prospective study was to examine the relationship between psychological stress and male reproductive function. The study population consisted of 450 men attending the infertility outpatient clinic and 45 men participating in an IVF-ICSI programme. Psychological stress was measured in four ways. Firstly, the "WHO (five) Well-Being Index" (1995 version) assessed the risk of depression in the previous two-week period. Secondly, Zung's Anxiety Scale Inventory was used to assess the features and severity of anxiety. Thirdly, the global reaction to various types of chronic life stress events related to family, partner, sexual behaviour, job strain and stress related to infertility treatment, with a more intense reaction corresponding to a higher score. Fourthly, the participant indicated by no $(=1)$ or yes $(=2)$ whether he had been exposed to the following acute 
stress situations: unemployment, moving house, personal illness or illness of a close relative, road accident. All questionnaires were completed after collection of the semen sample. The incidence of depression and anxiety symptoms in the two study populations $(6.5 \%$ and $5.3 \%$, and $20.3 \%$ and $30.8 \%$, respectively) was twice as high as that observed in the general population. Anxiety was more pronounced $(50 \%)$ in ICSI patients than in conventional IVF patients. To assess a relationship between psychological stress factors and male reproductive function, we used multiple regression analysis after adjustment of sperm parameters for age, sexual abstinence, type of infertility (primary or secondary), elevated body temperature, varicocele, and history of cryptorchidism and genital infection. Depression scoring was associated with increased sperm concentration (a $7.3 \%$ increase by score unit), an intense reaction to chronic stress was associated with a decreased sperm count ( $3.1 \%$ by score unit), and decreased motility "a", motility " $a+b$ ", and vitality $(0.5,0.5$, and $0.6 \%$ by score unit, respectively). Exposure to acute stress was associated with increased motility " $a+b$ " $(3.5 \%$ when the man was exposed to acute stress). Analysis using dichotomous division of fertile and infertile men did not reveal any significant correlation between any stress factor and infertility. These findings indicate that depression and anxiety are frequent in a population of infertile men attending the outpatient clinic or during IVF-ICSI attempts, while significant changes of sperm characteristics are related to depression (decreased sperm concentration), intense reaction to chronic stress (decreased sperm count, motility " $a$ " and " $a+b$ ", and vitality) and exposure to acute stress (better motility “a+b"). This study confirms that stress factors must be taken into account in the management of infertile couples with particular attention to depression and reaction to stress.

Key-words: acute stress, anxiety, chronic stress, depression, infertile men, sperm characteristics 